\title{
Application of Machine Vision Techniques in Textile (Fabric) Quality Analysis
}

\author{
Navneet Kaur ${ }^{1}$, Mandeep Dalal ${ }^{2}$ \\ ${ }^{1}$ (CSE Deptt., IITT College of Engg. \& Tech., Pojewal) Punjab, INDIA, \\ ${ }^{2}$ (CSE Deptt., IITT College of Engg. \& Tech., Pojewal) Punjab, INDIA,
}

\begin{abstract}
The textile quality can be determined by inspecting the texture in a cloth material. And thereby, this problem comes under the purview of texture analysis in machine vision system. Each texture analysis method presents a different potential for analysis of textured textile images. By considering their advantages and potential for successful flaw detection performance in homogeneous and jacquard textiles, machine vision system comes out to be a very handy method while determining the textile quality. Texture analysis refers to the characterization of regions in an image by their texture content. Texture analysis attempts to quantify intuitive qualities described by terms such as rough, smooth, silky, or bumpy as a function of the spatial variation in pixel intensities. In this sense, the roughness or bumpiness refers to variations in the intensity values, or gray levels.
\end{abstract}

\section{INTRODUCTION}

Most defects arising in the production process of a textile material are still detected by human inspection. The work of inspectors is very tedious and time consuming. They have to detect small details that can be located in a wide area that is moving through their visual field. The identification rate is about $70 \%$. In addition, the effectiveness of visual inspection decreases quickly with fatigue. However, the inspection process could be automated with machine vision techniques supported by defect identification algorithm. An image processing based machine vision system for visual inspection of fabric quality determination is proposed in the $\mathrm{p}$ [resented thesis work.

Digital image analysis permits a detailed analysis of basic structural parameters of linear textile products as thickness, hairiness and number of twists. Technique also enables the estimation of other characteristic features of the external structure of linear textile products, such as twist parameter and linear density coefficient. Defects can be classified as local or global. Global defects cause an overall distortion of the basic structure of the fabric and can be detected by means of Fourier analysis. Local

defects only affect a small area of the image of the fabric under inspection. In the proposed thesis work, it is proposed to develop an algorithm for detection of local as well as global defects from the fabrics online so as the corrective measure could be started at the time of detection of the defect.

\section{BRIEF LITERATURE SURVEY}

Texture analysis provides measures of properties such as smoothness, coarseness and regularity. There are three principal approaches in image processing are statistical, structural and spectral [6]. Statistical approaches yield characterization of textures as smooth, grainy and so on. Structural techniques deals with the arrangement of image primitives such as description of texture based on regularity spaced parallel lines [5,6]. Spectral techniques are based on properties of the Fourier spectrum and are used primarily to detect global periodicity in an image by identifying high energy, narrow peaks in the spectrum.
To address this problem, a need for the application of a comprehensive cojoint spatial-spatial frequency approach was identified [3]. A Gabor filter approach was chosen as a suitable representative of this class of techniques [1]. This research then successfully applied optimised 2-D Gabor filters to the textile flaw detection problem and provided a further support of their suitability for this task. A novel optimised 2-D Gabor algorithm presented in this study is an automatic solution which is adaptable to detect a large variety of textile flaw types, both structural and tonal [4].

\section{DEFECTS CLASSIFICATION}

In textile sectors, different types of faults are available i.e. hole, scratch, stretch, fly yarn, dirty spot, knot, slub, cracked point, misprints, color bleeding etc; if not detected properly these faults can affect the production process massively. Proposed textile analysis mainly detects four types of faults: hole, scratch, fresh as no fault and remaining faults as other fault. Inspection of $100 \%$ of fabric is necessary first to determine the quality and second to detect any disturbance in the weaving process to prevent defects from reoccurring.

\section{METHODOLOGY}

The digital analysis of two-dimensional images of fabric is based on processing the image acquirement, with the use of a computer. The image is described by a two-dimensional matrix of real or imaginary numbers presented by a definite number of bytes. The system of digital image processing may be presented schematically as shown in below Figure. The following operations are carried out during image quality improvement:

1. Image Acquisition

2. RGB to Gray Color Conversion

3. Image Enhancement (Thresholding)

4. Defect Identification and Texture Analysis

A Gabor filter scheme in the spatial domain following a fast pyramid implementation for computational efficiency is applied for texture analysis. An image with the joint contribution of the complete set of multi-resolution and 
multi-orientation channels is binarized. In the binary output image local defects appear segmented from the background. The only considerations that require attention are optical conditions such as lightness and scale to guarantee optimal performance, and a preliminary analysis of a prototype defect-free sample to extract the mean and standard deviation of its texture descriptors.

\section{IMAGE CAPTURING}

Textile/fabric surface image is acquired by using the CCD camera from top of the surface from a distance adjusted so as to get the best possible view of the surface. Below figures show the quality of the acquired fabric images. The textile images under test are of size $256 \times 256$ (64KB). For proper imaging, uniform lighting system is to be maintained to avoid any illusive defect by virtue of light reflection properties falling on surface.

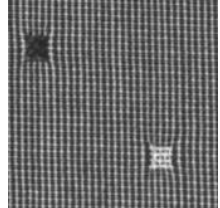

Fig. 1

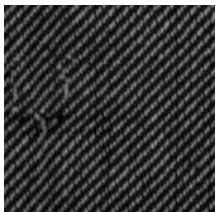

Fig. 2

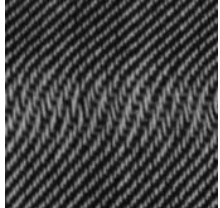

Fig. 3
Different Fabric/Textile Images

Originally, the images are acquired at RGB color scale. The images then are converted to gray scale using rgb2gray function in matlab.

\section{IMAGE THRESHOLDING}

Histogram equalization method is adopted to enhance the contrast of the fabric surface. Histogram Equalization algorithm works good in this case as the fabric texture. Below figures show the result after Histogram equalization algorithm for thresholding.

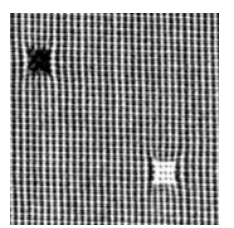

Fig. 4

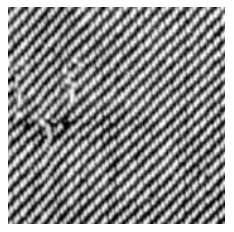

Fig. 5

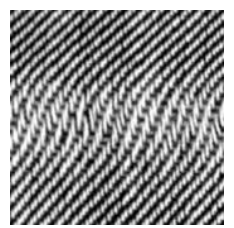

Fig. 6
Images after Histogram Equalization algorithm

Fig. 4, 5 and 6 show the after application of the Histogram Equalization algorithm. Fig. 7, 8 and 9 show the histogram of the above respective images before and after histogram equalization.
Histograms Before Hist. Eq. $\quad$ After Hist. Eq.
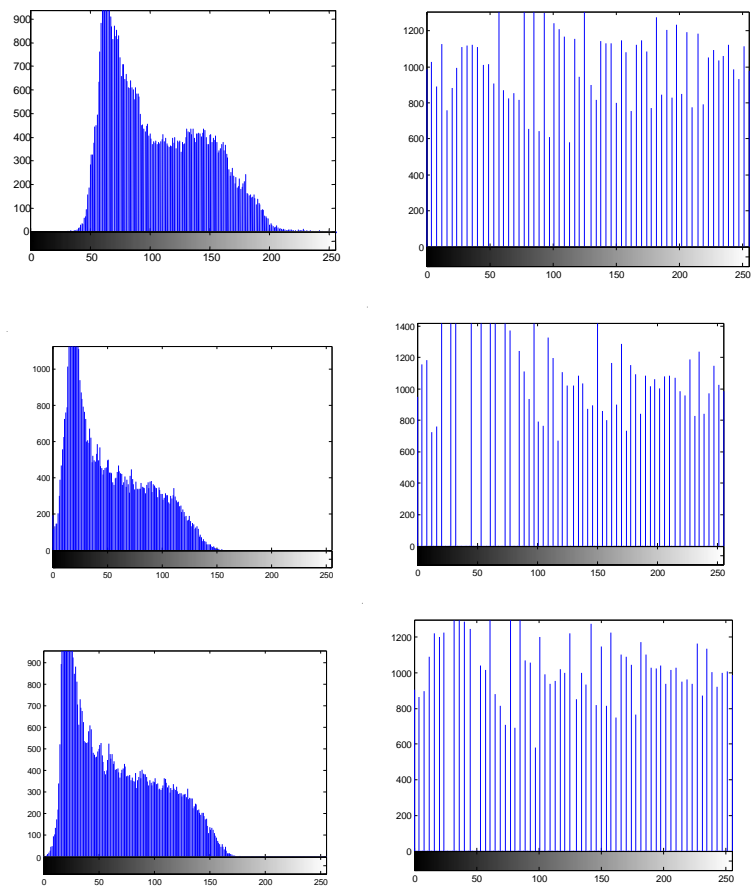

\section{EXPERIMENT AND RESULTS}

Following test image of a defected textile surface has been used for defect identification. The image has been exposed to histogram equalization algorithm for thresholding. The thresholded image is brought under noise removal program, where the uneven weaving is detected as spots shown in fig. 9. Fig. 7 and 8 shows the original and thresholded images.

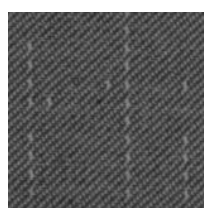

Fig. 7

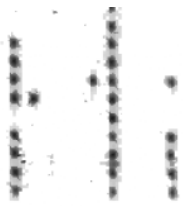

Fig. 8

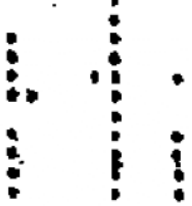

Fig. 9
Fig. 7, 8 and 9 shows the processing of the multi-thread broken type defect.

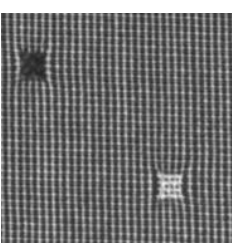

Fig. 10
Fig. 11

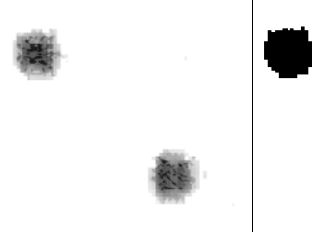

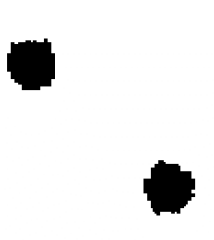

Fig. 12
Fig. 10, 11 and 12 present the large defects of different colors in a plain fabric with black and white threads. 


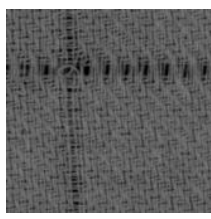

Fig. 13

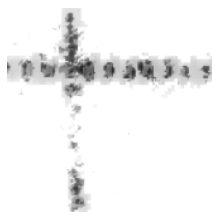

Fig. 14
Fig. 15

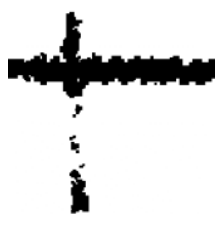

Fig. 13, 14 and 15 indicate white twill fabric with crossed break of multiple threads.

In case of textile/fabric analysis using machine vision system, we are primarily concerned about the defect identification and which may be on account of uneven weaving from the normal weaving pattern or color difference (fading) or any other defect. Once the defect is identified, corrective action could be initiated. But, main emphasis is on getting alert as soon as the defect is identified.

\section{Conclusion}

The method proposed for local defect detection is a useful tool for inspecting industrial materials with periodic regular texture. As we intended, a general improvement and enlargement of the vision system capabilities can be achieved by using the proposed algorithm to detect local defects in regular textures. In the binary output image local defects appear segmented from the background. One of the most important advantages of the method is that it is multipurpose without requiring any adjustment. Furthermore, it can be applied to composite patterns with elements of different brightness without any particular adaptation. The versatility of the method has been demonstrated not only by its applicability to different regular textures but also, for a given texture, the method allows to detect a variety of defects. The method does not need human supervision nor previous knowledge about the texture or defect.

\section{ACKNOWLEDGEMENTS}

We are thankful to Mr. Vikas Goel, Sr. Project Manager, CDAC, Mohali, Punjab for his valuable guidance and continuous support in making this paper.

\section{REFERENCES}

[1] Escofet J., Millán M.S., Ralló M., "Modelling of woven fabric structures based on Fourier image analysis", Appl. Opt. 40, 34, 6170-6176 (2001).

[2] Hu M.C., Tsai I.S. "Fabric Inspection Based on best Wavelet Packet Bases", Textile Res. J. 70(8), 662670 (2000)..

[3] Kang T.J. et al. "Automatic Structure Analysis and Objective Evaluation of Woven Fabric Using Image Analysis", Textile Res. J. 71(3), 261-270 (2001)

[4] Weldon T. P., Higgins W. E., Dunn D. F., "Gabor filter design for multiple texture segmentation", Opt. Eng. 35(10), 2852-2863 (1996).

[5] Tsai I.S., Hu M.C. "Automatic Inspection of Fabric Defects Using an Artificial Neural Network Technique", Textile Res. J. 66(7), 474-482 (1996)
[6] Portilla J., Navarro R., Nestares O., "Texture synthesis-by-analysis method based on a multiscale early-vision model”, Opt. Eng. 35(8), 2403-2417 (1996).

[7] R anden T., Hakon-Husoy J., "Multichannel filtering for image texture segmentation", Opt. Eng. 33 (8), 2617-2625 (1994). 